\title{
A Novel Counter Sheet-flow Sandwich Cell Culture Device for Mammalian Cell Growth in Space
}

\author{
Shujin Sun • Yuxin Gao • Nanjiang Shu • \\ Zemei Tang $\cdot$ Zulai Tao $\cdot$ Mian Long
}

Received: 30 December 2006 / Accepted: 18 October 2007 / Published online: 16 April 2008

(C) Springer Science + Business Media B.V. 2008

\begin{abstract}
Cell culture and growth in space is crucial to understand the cellular responses under microgravity. The effects of microgravity were coupled with such environment restrictions as medium perfusion, in which the underlying mechanism has been poorly understood. In the present work, a customer-made counter sheet-flow sandwich cell culture device was developed upon a biomechanical concept from fish gill breathing. The sandwich culture unit consists of two side chambers where the medium flow is counter-directional, a central chamber where the cells are cultured, and two porous polycarbonate membranes between side and central chambers. Flow dynamics analysis revealed the symmetrical velocity profile and uniform low shear rate distribution of flowing medium inside the central culture chamber, which promotes sufficient mass transport and nutrient supply for mammalian cell growth. An onorbit experiment performed on a recovery satellite was used to validate the availability of the device.
\end{abstract}

S. Sun $\cdot$ Y. Gao $\cdot$ N. Shu $\cdot$ Z. Tang $\cdot$ Z. Tao $\cdot$ M. Long

National Microgravity Laboratory, Institute of Mechanics,

Chinese Academy of Sciences,

Beijing 100190, People's Republic of China

S. Sun $\cdot$ Y. Gao $\cdot$ N. Shu $\cdot$ Z. Tao $\cdot$ M. Long

Center for Biomechanics and Bioengineering,

Institute of Mechanics, Chinese Academy of Sciences,

Beijing 100190, People's Republic of China

S. Sun $(\bowtie) \cdot$ M. Long

National Microgravity Laboratory and Center of Biomechanics and Bioengineering, Institute of Mechanics,

Chinese Academy of Sciences,

Beijing 100190, People's Republic of China

e-mail: sunshj@imech.ac.cn

M. Long

e-mail: mlong@imech.ac.cn
Keywords Counter sheet-flow sandwich cell culture . Mammalian cell growth $\cdot$ Microgravity

\section{Introduction}

Cellular responses under microgravity are crucial to understand the biological and physiological processes in space. To date little has been known about the mechanism how cells (especially mammalian cells) response to microgravity. Biological responses of cells in space depend strongly on how cells are cultivated on orbit, because the experimental conditions under microgravity are notably different from conventional laboratory conditions on ground. In microgravity environment, buoyancy and convection tend to disappear due to weightlessness, and the pattern of mass transport and nutrient supply for cells changes dramatically. Basically, mass exchange is depressed under microgravity, which influences the proliferation, differentiation, metabolism, and apoptosis of cells (Kacena et al. 2003; Lewis et al. 1998; Crawford-Young 2006). Furthermore, many conventional assays in cell biology on ground are no longer available in space.

Cell growth requires the sufficient mass transport and nutrient supply. For maintaining nutrient supplies and removing metabolic wastes, for example, refreshing the culture medium is a simple procedure on ground-based conditions. In space, however, the procedure becomes complicated and hard to perform, since it should be done in a closed-loop system automatically. Continuous perfusion of culture medium is required to maintain cellular metabolism, in which shear stress induced by medium flow may have the impacts on cell viability and function (Begley and Kleis 2000; Mardikar and Niranjan 2000; Papadaki et al. 1996; Imberti et al. 2002). For example, shear stress 
affected endothelial cell proliferation and the cells were oriented along flow direction (Imberti et al. 2002). Besides, gas exchange also becomes complicated in space because of weightlessness and lacking the inconsumable atmosphere.

When perfusing culture medium, cellular responses in space are manipulated by combined effects of microgravity, shear flow, and other flow dynamic factors. At this sense, it is hard to isolate the effect of microgravity from that of shear flow. In the pioneering space cell biology experiments and other experiments in clinostat, the results reported have often proved to be inconsistent (Crawford-Young 2006; Sonnenfeld 2002), probably due to inadequately controlled experimental conditions. Another line of reasoning may come from negative impact of high shear on cell growth, even though the high shear may be required to suspend cells in the medium. To understand the mechanism how microgravity affects cell responses in space, flow dynamics and mass transport analysis inside the culture system or cell bioreactor are required to distinguish the direct and indirect effects of microgravity on cell growth and proliferation.

Developing advanced device and instrumentation in space cell biology has high priority in the new century (Martin and Vermette 2005; Freed and Vunjak-Novakovic 2002). Although several types of space cell bioreactors for cell species from bacteria to mammalian cells have been developed and utilized in past decades (Martin and Vermette 2005; Freed and Vunjak-Novakovic 2002; Dedolph and Dipert 1971; Walther 2002; Misener et al. 2000; van der Schoot et al. 2001), environment restrictions from experimental devices (so-called habitat effects), especially for culturing mammalian cells in space, are still open issues to be addressed, since mechanical effects seem to be critically important but poorly understood. In addition, the payload resources (e.g., size, weight, and energy consumption) in spacecraft are costful and limited, which has to be optimized in developing a space cell bioreactor. In this study, a novel counter sheet-flow sandwich cell culture (CSSCC) device was designed upon a biomechanical principle from fish gill breathing with well-controlled flow dynamics and mass transport. A recoverable satellite passenger payload experiment was performed to validate the accessibility and reliability of CSSCC device.

\section{Biomechanical Principle}

Basically, the growth and proliferation of mammalian cells in space requires flow-induced culture medium supply, temperature stability, $\mathrm{pH}$ control, and sufficient gas exchange in an airproofed bioreactor. All the factors were integrated into designing the novel sandwich culture unit.
To provide sufficient mass transport and nutrient supply in a space cell bioreactor, one of approaches is to perfuse the culture medium continuously through cell culture chamber. This induces the concentration gradient of nutrient substances and metabolic wastes along the flow direction, which provides inhomogeneous distribution of mass exchange in cell culture zone. The biomechanical principle in the current study was based on a bionic phenomenon of fish gill breathing. Fish gills are composed of filaments which has many tiny folds (so-called lamellae). Blood flow in the arterioles that run inside the filaments and lamellae is counter-directional from the water passing the gills (Piiper and Baumgarten-Schumann 1968a; Hughes 1972). In a uni-directional flow (Fig. 1a), the available oxygen uptake is only $\sim 50 \%$ by blood from the water where oxygen in the blood and water reaches an equilibrium, and further diffusion of oxygen no longer takes place beyond the saturated uptake. In a counter-directional flow (Fig. 1b), however, oxygen gradient along two counter

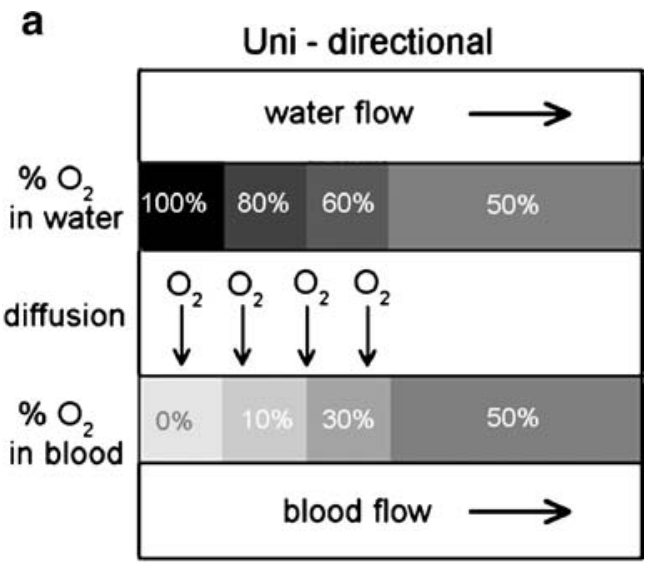

b

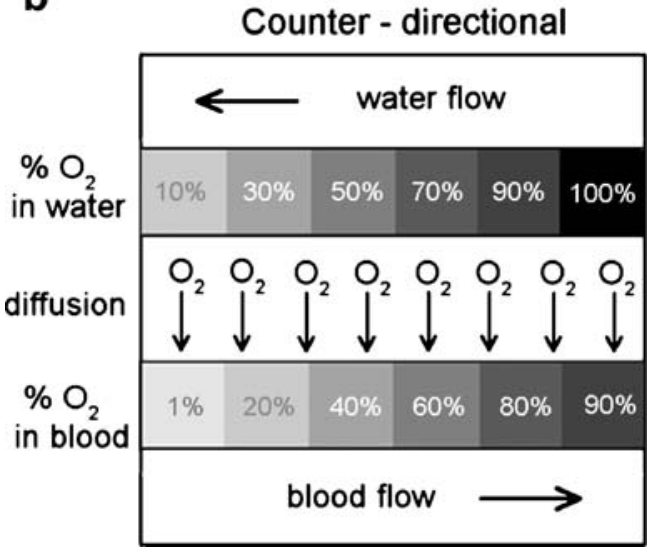

Fig. 1 Schematic to demonstrate the concept of novel CSSCC device. a Only $\sim 50 \%$ oxygen uptake is available by blood from the water where oxygen in the blood and water reaches an equilibrium and further diffusion of oxygen no longer takes place beyond the saturated uptake in a uni-directional flow. b Oxygen gradient between two counter directions is maintained and diffusion of oxygen remains available beyond $50 \%$ uptake, which enhances the oxygen exchange up to $80-90 \%$ in a counter-directional flow 
directions is maintained and diffusion of oxygen remains available beyond $50 \%$ uptake, which enhances the oxygen exchange up to $80-90 \%$ (Piiper and Baumgarten-Schumann 1968b; Piiper et al. 1986). The underlying mechanism in the oxygen-blood exchange provides a conceptual framework for our design.

To provide uniform and sufficient mass transport and nutrient supply, a sandwich culture unit was designed (Length:Width:Height $=$ 53:30:2.7 mm (inner dimensions)), which includes a central culture chamber with a thickness of $2.0 \mathrm{~mm}$, and two adjacent medium perfusion chambers (side chambers) with a thickness of $0.35 \mathrm{~mm}$ each (Fig. 2). The three chambers were separated by two porous polycarbonate (PC) membranes (with a nominal pore diameter of $1.0 \mu \mathrm{m}$ and a thickness of $12 \mu \mathrm{m}$ ), by which the cells were contained within culture chamber and through which the medium can be infiltrated into culture chamber from side chambers. Each of side chambers has its own inlet and outlet. Culture medium was driven by a peristaltic pump into side chambers in the opposite directions, thereby forming a counter sheet-flow cell culture unit (Fig. 2).

\section{Flow Dynamics Analysis}

To predict the perfusion of culture medium in the culture unit, flow dynamics analysis was done to quantify the velocity profile and shear rate distribution inside the unit. Finite element analysis was accomplished by using commercial CFD software of FLUENT 6.1. In a typical two dimensional numerical calculation upon the assumption that no cells are present in the culture chamber, the heights of side and culture chambers are given respectively at 0.35 and $2.0 \mathrm{~mm}$, and the length of the chambers is given at $30 \mathrm{~mm}$. The influx velocity in side chamber is set at $2 \times$ $10^{-5} \mathrm{~m} / \mathrm{s}$. A porous jump model was used to simulate the PC membrane where the membrane was assumed to be an isotropic porous media. No no-slip boundary condition at the membrane was given in the calculation, since tangential velocity on the non-pore zone and normal velocity on the pore zone at the membrane should follow flow continuity law. In FLUENT software, the model defines the pressure change across the membrane as a combination of Darcy's Law and an additional inertial loss term:

$\Delta p=-\left(\frac{\mu}{\alpha} v+C_{2} \frac{1}{2} \rho v^{2}\right) \Delta m$,

where $\alpha$ is the permeability of the membrane, $\mathrm{C}_{2}$ is the pressure-jump coefficient, and $\Delta m$ is the thickness of the membrane. In the numerical calculation, only viscous resistance coefficient was taken into account because the inertial resistance term is several orders of magnitude smaller than the viscous term. As such, the porous jump model reverts to Darcy's Law where only $\alpha$ and $\Delta m$ need to be given. Here the thickness of the membrane $\Delta m$ is $12 \mu \mathrm{m}$, and the permeability coefficient $\alpha$ can be estimated from the mean pore diameter and density of the membrane ( $1 \mu \mathrm{m}$ and $2 \times 10^{7}$ pores $/ \mathrm{cm}^{2}$, respectively). A packed bed model was used to estimate the permeability coefficient $\alpha$. Briefly, the mean particle diameter $\left(D_{p}\right)$ of the packed bed was estimated by converting void fraction of the membrane into solid fraction, and then $\alpha$ was calculated, upon an empirical Ergun equation ( $\alpha$ is the function of $D_{p}$; refer to FLUENT User's Guide), to be $\alpha=1.45 \times 10^{-16} \mathrm{~m}^{2}$. Systematically-varied $D_{p}$ was also tested and no significant differences were found in the calculations.

As exemplified in Fig. 3, flow of culture medium was symmetrically distributed inside the three chambers, which, as compared to fish gills in a counter-direction perfusion, enabled to reduce the gradient of mass transport and nutrient supply along flow direction in the culture zone. In addition, flow in the culture chamber was quite slow, and reached a minimum of nearly zero in the central area. This was important since such a slow flow inside the culture chamber promoted sufficient mass transport, nutrient supply, and gas exchange, and was propitious to minimizing the habitat effects.
Fig. 2 Schematic for set-up of CSSCC device. Culture medium was pumped to flow through bioreactor unit in two counterdirections from medium containing bag to medium collecting bag. Cells were seeded onto the multi-pored slice mounted at the central position of cell culture unit
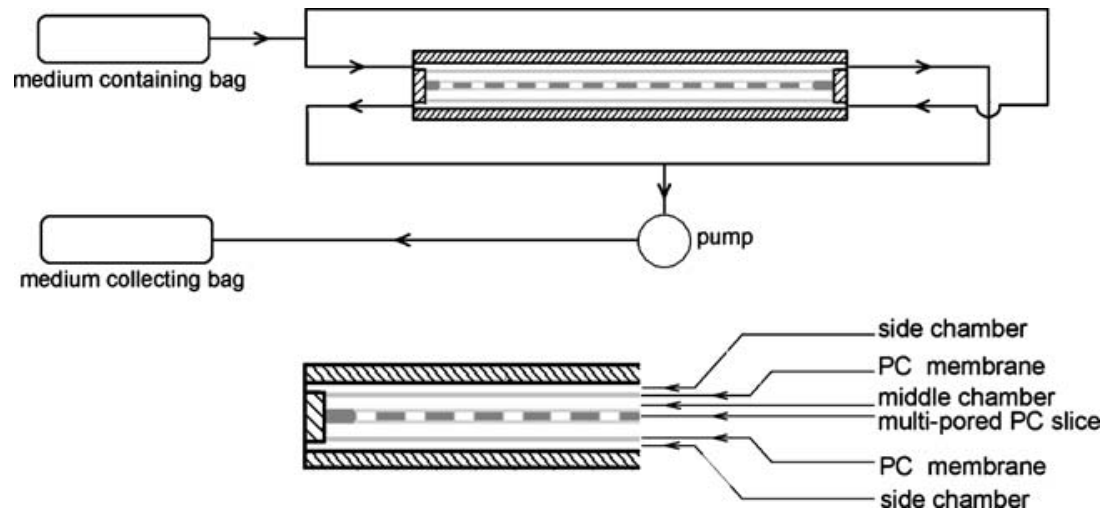
Fig. 3 Velocity profile of flowing medium at the middle cross-section of CSSCC device by plotting the velocity vectors colored by velocity magnitude. Only half of flow field was presented upon geometric symmetry for clarity
$2.57 \mathrm{e}-05$
$2.30 \mathrm{e}-05$
$2.03 e-05$
$1.76 e-05$
$1.49 \mathrm{e}-05$
$1.22 \mathrm{e}-05$
$9.49 e-06$
$6.78 e-06$
$4.07 e-06$
$1.36 \mathrm{e}-06$

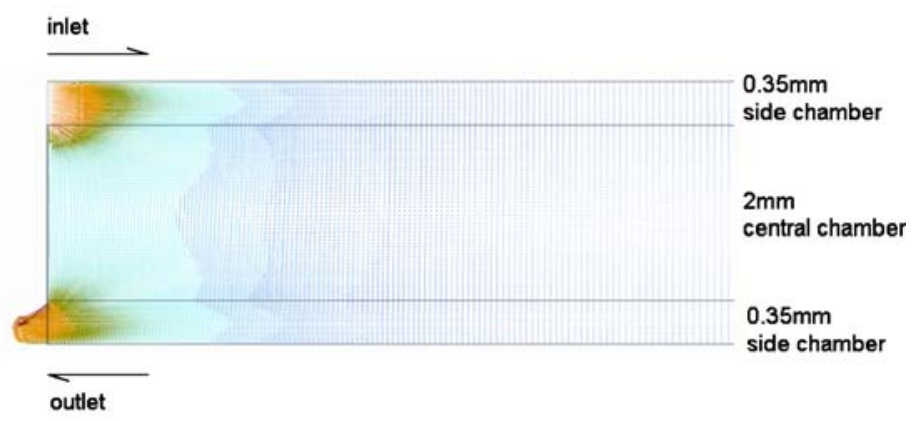

Velocity Vectors Colored By Velocity Magnitude (m/s)

Flow shear rate was also quantified from flow dynamics analysis. In the typical calculation as exemplified in Fig. 4, the shear rate at the most locations of central chamber is less than $0.1 \mathrm{~s}^{-1}$ at a flow rate of $1.15 \mathrm{ml} / \mathrm{h}$ except at the vicinity of inlet or outlet. This translated to a shear stress of $10^{-4} \mathrm{~N} / \mathrm{m}^{2}$ at the medium viscosity of $10^{-3} \mathrm{~Pa} \cdot \mathrm{s}$, which was one order of magnitude lower of critical shear stress to affect cell growth (Begley and Kleis 2000). Taken together, such a medium flow provided sufficient mass transport and nutrient supply at low shear rate.

\section{CSSCC Set-up}

The CSSCC device was composed of a cell culture unit, a flow control unit, a temperature control unit, a signal input/ output unit, and a system case.

Cell culture unit was constructed using commercial PC plates. Two porous PC membranes were used as the permeable membranes to separate the side and central chambers. A multi-pored PC slice $(0.6-0.8 \mathrm{~mm}$ in thickness), treated as hydrophilic with oxygen plasma, was embedded at the central position of the culture chamber as the substrate for cell seeding (total seeding area $\sim 28 \mathrm{~cm}^{2}$ ) (Fig. 2). All perfusing tubes and medium containing and collecting bags were made of medical silicone rubber. PC plates, membranes and slices, and silicone tubes and bags were autoclaved before seeding the cells. Perfusion of culture medium was manipulated using a micro peristaltic pump (P900, Instech Laboratories, Inc., USA). Temperature inside the device was regulated at $36.5 \pm$ $1{ }^{\circ} \mathrm{C}$ using a Eurotherm 2132 miniature temperature controller (Eurotherm Limited) and PT-100 platinum resistance thermometers, together with heating membranes, a mini-fan, and heatproof materials stuck onto inner wall of the case. Medium $\mathrm{pH}$ value $(\mathrm{pH}=7.2)$ was pre-set by filling standard air mixed with $5 \% \mathrm{CO}_{2}$ into the tank before it was closed up for launch, in which standard air was used to provide sufficient oxygen supply and $\mathrm{CO}_{2}$ was used to prevent culture medium from alkalization via permeable membranes and bags. The amount of standard air mixed with $5 \% \mathrm{CO}_{2}$ was calculated upon the given flight duration and was tested by a ground-based control experiment. All units were integrated into an aluminium alloy case and sealed with $\mathrm{O}$ -
Fig. 4 Shear rate distribution at the middle cross-section of CSSCC device by plotting the velocity vectors colored by shear rate magnitude. Only half of shear rate field was presented upon geometric symmetry for clarity

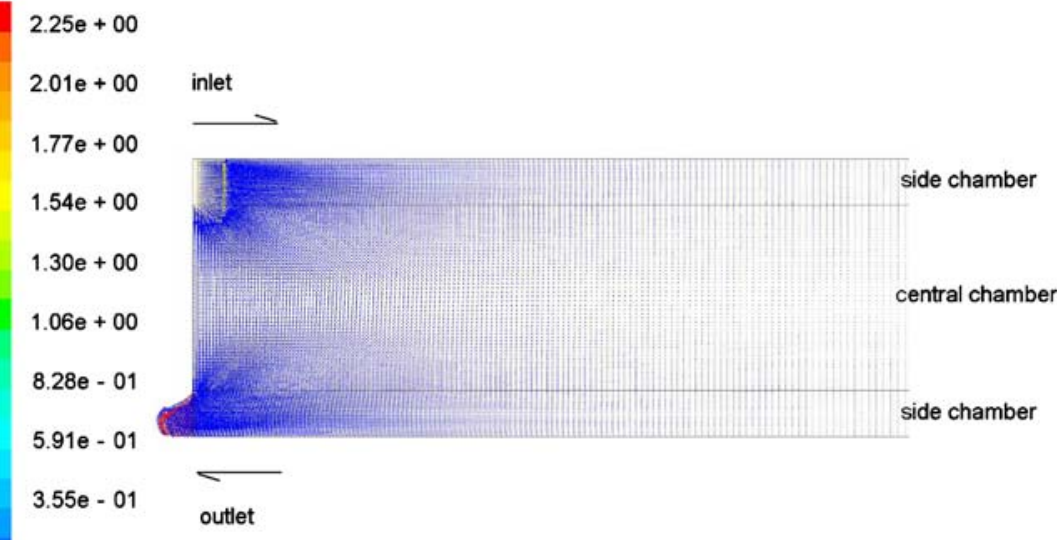

Velocity Vectors Colored By Strain Rate (1/s) 
shaped rubber seal rings and seal sockets. The vacuum leak test was performed for the device with gas leakage rate of less than $0.1 \%$ per hour.

\section{CSSCC Validation on Payload Experiment}

A trophoblastic tumor cell line (JAR) (ATCC, Rockville) was used in the satellite payload experiment launched in August, 2005 to test the availability of CSSCC device. JAR cells were seeded at 32-h before launching, and the CSSCC device was connected with power supply to provide the pre-flow of culture medium. After 3-h pre-culture, standard air mixed with $5 \% \mathrm{CO}_{2}$ was filled into the tank, and the device was then sealed and mounted on the satellite. Flow rate was controlled to be $1.15 \mathrm{ml} / \mathrm{h}$ for each side chamber. This rate came from estimating oxygen uptake and nutrient consumption upon oxygen solubility of $0.26 \mathrm{mM} / \mathrm{L}$ in water and oxygen consumption rate of $0.006-0.3 \mu \mathrm{M} / 10^{6}$ cells/h for mammalian cells (Eyer et al. 1995; Fleischaker and Sinskey 1981), as well as maximum culture medium volume of $1.2 \mathrm{ml}$ for $10^{6}$ cells per hour. The on-orbit experiment was performed for a 4-day (96-h) run out of 18day flight duration, and space media samples were collected from the collecting bag and the two side chambers after the satellite was recovered. Our tests to recovered CSSCC device indicated that the device worked well with a designed flow at pre-set temperature and $\mathrm{pH}$ value. It was also evident that the culture medium was collected properly, as expected. Such the payload experiment validated the accessibility and reliability of CSSCC device.

\section{Discussion}

The goal of the current work is to develop a novel cell culture system used to perform mammalian cell culture in space with sufficient mass transport and nutrient supply. Upon a biomechanical concept from flow of fish gills (Fig. 1), a CSSCC device was optimistically constructed by taking into account the limited resources for spacecrafts (Fig. 2). Flow dynamics analysis demonstrated the symmetrical velocity profile and low shear rate distribution (Figs. 3 and 4), which was used to predict mass transport and nutrient supply and to minimize the habitat effects in space cell culture.

A reliable, efficient, and effective hardware is crucial to unravel cellular responses in space. One of key issues for designing such the space cell culture unit is how to distinguish the direct and indirect effects of microgravity when providing sufficient mass transport and nutrient supply by a perfusing flow. Even though there is still a long journey in both hardware design and experimental scheme to isolate such the direct effect, the conceptual framework reported provides a basic idea that controllable flow of culture medium is pre-requisite, as was observed in Figs. 3 and 4. It was also demonstrated that sufficient flow and low shear rate (or shear stress) inside the culture chamber has to be balanced to avoid mechanical injury on growing cells. Flow dynamics analysis reported neglected the existence of cells cultured inside the chamber, which even simplified the numerical calculation but still provided a basic idea for flow field pattern. Further flow analysis is required by taking into account the existence of culture cells.

Cell culture in a controllable environment is a challenging field since there are many factors to affect the biological process. For example, the effects of mechanical vibration and acceleration and deceleration during launching and landing of satellite were still unknown. Cells and culture medium were kept on orbit for additional 2 weeks without lowtemperature protection and fixation since only 4-day on-orbit experiment out of 18-day flight duration was payload available. Gas exchange was unable to be monitored in situ when cells were grown on orbit. Major reasoning for the issues raised above was attributed to the limitation of payload volume $(220 \times 180 \times 100 \mathrm{~mm})$, weight $(4.6 \mathrm{~kg})$, and power supply $(16 \mathrm{~W})$. Nevertheless, miniaturizing the bioreactor, lowering the power supply, monitoring the $\mathrm{pH}$ value of medium in situ, and visualizing the cell growth are required for further optimizing the CSSCC device. And more cell species and relevant biological responses need to be tested on different flow parameters and culture unit geometry to further validate the availability of the device.

\section{Conclusion}

The present work not only developed the rational for the novel CSSCC system upon a biomechanical concept but also validated the availability of the system upon an onorbit experiment. To minimize the habitat effects on cell culture in space, both mechanical- and biological-based designs are important to cell culture device or cell bioreactor.

Acknowledgements We thank Shiqi Wang, Hong Li and Dr. Xiaonan Hou for technical assistances. We also thank Drs. Yiran An and Jiaquan Wang for useful discussions. This work was supported by Chinese Academy of Sciences grants KJCX2-SW-L06 and 20051-16, and National Science Foundation of China grants 10332060 and 30225027 .

\section{References}

Begley, C.M., Kleis, S.J.: The fluid dynamic and shear environment in the NASA/JSC rotating-wall perfused vessel bioreactor. Biotechnol. Bioeng. 70, 32 (2000) 
Crawford-Young, S.J.: Effects of microgravity on cell cytoskeleton and embryogenesis. Int. J. Dev. Biol. 50, 183 (2006)

Dedolph, R.R., Dipert, M.H.: The physical basis of gravity stimulus nullification by clinostat rotation. Plant Physiol. 47, 756 (1971)

Eyer, K., Oeggerli, A., Heinzle, E.I.: On-line gas analysis in animal cell cultivation. II. Methods for our measurements and its application for control. Biotechnol. Bioeng. 45, 54 (1995)

Fleischaker, R.J., Sinskey, A.: Oxygen demand and supply in cell culture. Eur. J. Appl. Microbiol. Biotechnol. 12, 193 (1981)

Freed, L.E., Vunjak-Novakovic, G.: Spaceflight bioreactor studies of cells and tissues. Adv. Space Biol. Med. 8, 177 (2002)

Hughes, G.M.: Distribution of oxygen tension in the blood and water along the secondary lamella of the icefish gill. J. Exp. Biol. 56, 481 (1972)

Imberti, B., Seliktar, D., Nerem, R., et al.: The response of endothelial cells to fluid shear stress using a co-culture model of the arterial wall. Endothelium 9, 11 (2002)

Kacena, M.A., Todd, P., Landis, W.J.: Osteoblasts subjected to spaceflight and simulated space shuttle launch conditions. In Vitro Cell. Dev. Biol. Anim. 39, 454 (2003)

Lewis, M.L., Reynolds, J.L., Cubano, L.A., et al.: Spaceflight alters microtubules and increases apoptosis in human lymphocytes (Jurkat). FASEB J. 12, 1007 (1998)

Mardikar, S., Niranjan, K.: Observations on the shear damage to different animal cells in a concentric cylinder viscometer. Biotechnol. Bioeng. 68, 697 (2000)
Martin, Y., Vermette, P.: Bioreactors for tissue mass culture: Design, characterization, and recent advances. Biomaterials 26, 7481 (2005)

Misener, L., Sindrey, D., Smith, T., et al.: Development of a microgravity cell culture platform for the study of bone cell metabolism.In: 16th Annual Meeting of American Society for Gravitational and Space Biology (ASGSB-CSA-ELGRA Combined Meeting); 25-28 October 2000; Montréal, QC, Canada

Papadaki, M., McIntire, L., Eskin, S.: Effects of shear stress on the growth kinetics of human aortic smooth muscle cells in vitro. Biotechnol. Bioeng. 50, 555 (1996)

Piiper, J., Baumgarten-Schumann, D.: Transport of $\mathrm{O}_{2}$ and $\mathrm{CO}_{2}$ by water and blood in gas exchange of the dogfish Scyliorhinus stellaris. Respir. Physiol. 5, 326 (1968a)

Piiper, J., Baumgarten-Schumann, D.: Effectiveness of $\mathrm{O}_{2}$ and $\mathrm{CO}_{2}$ exchange in the gills of the dogfish (Scyliorhinus stellaris). Respir. Physiol. 5, 338 (1968b)

Piiper, J., Scheid, P., Perry, S.F., et al.: Effective and morphometric oxygen-diffusing capacity of the gills of the elasmobranch Scyliorhinus stellaris. J. Exp. Biol. 123, 27 (1986)

Sonnenfeld, G.: The immune system in space and microgravity. Med. Sci. Sports Exerc. 34, 2021 (2002)

van der Schoot, B., Boillat, M., de Rooij, N.: Micro-instruments for life science research. IEEE Trans. Instrum. Meas. 50, 1538 (2001)

Walther, I.: Space bioreactors and their applications. Adv. Space Biol. Med. 8, 197 (2002) 